\title{
Performance Evaluation of OLSR Routing Protocol using Different Mobility Models in MANETS
}

\author{
Mubarak Mohammed Al-Ezzi Sufyan \\ MS(IT) Research Scholar IBMS \\ The University of Agriculture, Peshawar-Pakistan
}

\begin{abstract}
The performance of a protocol in MANETs can be measured using some parameters, which also includes simulation environment like campus or military. This research is done in the campus environment using the simulation parameters such as area size, packet size, time of simulation, UDP connection...etc for measuring the performance matrices such as sent, received and dropped rate of CBR data packets, average end-to-end delay, packet delivery ratio, normalized routing overhead and average throughput in order to evaluate the performance of OLSR routing protocol using different mobility models, namely, Random-Based Mobility Model (RBMM) and Random WayPoint Mobility Model (RWMM) in MANET by using NS-2.35 simulator. NS-2.35 is an open source simulation software and the simulation of this research include eight scenarios dependent on mobility model based on the number of nodes such as 40,60,80 and 100 mobile nodes and then using AWK, Xgraph and Trace_graph for analyzing data and showing graph. Based on obtained results the performance of OLSR by using RWPMM is more efficient as compared to the performance of OLSR under RBMM, because maximum parameters gave better result with RWPMM as compared to with RBMM.
\end{abstract}

\section{Keywords}

MANETs, OLSR, Multi Point Relays (MPR), Mobility Models, RWPMM, RBMM.

\section{INTRODUCTION}

\subsection{Mobile Ad Hoc Networks (MANETs)}

MANETs is a temporary network without any infrastructure and centralized administration, self-configuring (intelligent nodes), multi-hop and systematic devices communicating with both dynamically within their radio frequency range and also with those which are outside the range during hops by using route discovery mechanisms (connected by wireless link) anytime, anywhere with anyone unlike mobile telecommunication for calling [1]. There are more features of MANETs like easy deployment, easy joining \& leaving the network, mobility, multi-hop routing, dynamic topology, multi-casting, uni-casting, each node can work as a host as well as a router, autonomous terminal and distributed operation. There are some challenges in MANETs like topology changes, bandwidth and energy consumption, broadcasting, security and reliability, quality of service (QoS), Inter-networking, routing... etc. [2].

\author{
Khalid Saeed \\ Lecturer Computer Sciences IBMS \\ The University of Agriculture, Peshawar-Pakistan
}

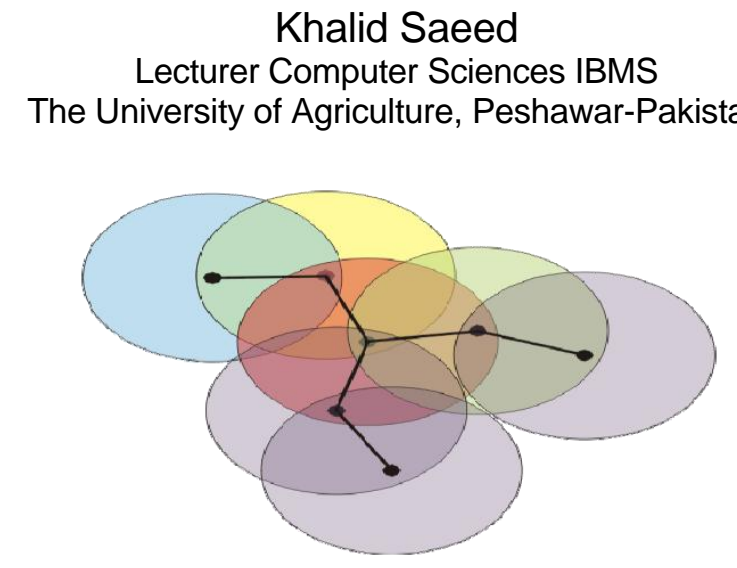

Fig 1. MANETs Structure

\subsection{MANETs Routing Protocols}

MANETs routing protocols are standards for controlling how nodes select the route for transmission packets between the source node and the destination node. The classification of MANETs routing protocols are divided into three categories such as: Reactive Routing Protocols (On-Demand), Proactive Routing Protocols (Table-Driven) and Hybrid Routing Protocols [3]

\subsection{OLSR Routing Protocol}

The Optimized Link State Routing (OLSR) protocol is a linkstate proactive routing protocol, which was developed by INRIA (France) ["Jacquet", 2001] RFC 3626. OLSR operates as a proactive routing protocol (table-driven) of the classical link state algorithm that is a topology-based, because it exchanges information of the topology of the network with neighbor nodes and shares routing table information periodically. It is a better protocol as compared to other protocols, because it is used for large and dense networks and it works independently from other protocols. OLSR has four kinds of control messages such as:

1. HELLO Message is generated by each node for neighbor's link sensing and MPR selection. It contains its own address and the list of its 1-hop neighbors by exchanging HELLO messages (HELLO INTERVAL is sent after every 2 seconds).

2. Topology Control (TC) message is generated only by MPR nodes to advertise MPR selector information about the "topology" of the network by giving each neighbor selector sequence number incremented by one. It contains a list of the sender's MPR selector sender by TC messages periodically (TC INTERVAL is sent after every 5 seconds).

3. Multiple Interface Declaration (MID) message is performing the task of declaring the presence of multiple interfaces on a node, because each node in the network has IP address, so it contains a list of the node's IP addresses in the network for transmitting these messages on more than one interface. 
4. Host and Network Association (HNA) message is used for association between OLSR network and other network via the node as a gateway, which is advertising OLSR network information to other networks [4].

\subsection{Multi-Point Relay (MPR)}

The responsibility of MPR is forwarding control traffic, declaring link state information and reducing the number of retransmission required by flooding control traffic to provide an efficient mechanism, so that each node selects its MPR set from among its 1-hop symmetric neighbors. The "MultiPoint Relay (MPR) set" for any node in the network is a set of nodes selected by that node from Symmetric 1-hop neighborhood, which retransmits its message to that node [4].

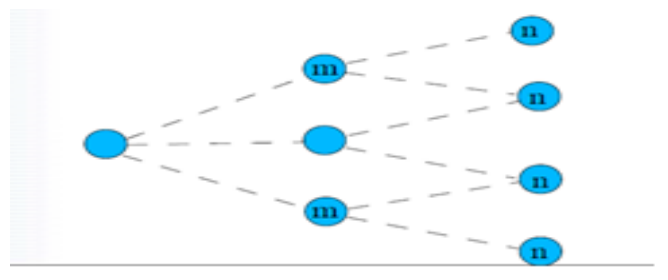

Fig 2. MPR Selection

\subsection{Mobility Models}

Mobility models are mathematical algorithms, which have been designed in order to try to model the behavior of real movement pattern of mobile users in geographic location and speed. A mobility model is also used for performance evaluation in many simulations to represent the realistic movements of nodes in simulation. In [5] the mobility models are classified into two main categories which are as follows:

\subsubsection{Group Mobility Models}

In this category, the nodes are divided into many group, each group consists of one node header and other nodes follow, so that each group will trigger movement both dependent on behavior of their header by different parameters such as new position, pause time, speed ...etc i.e. each group of soldiers in a military. This model is divided into the following sub models: Reference Point Group, a set of spatially correlated models ...etc.

\subsubsection{Entity Mobility Models}

In this category, each node movement is independent of each other under different impact parameters such as target, pause time, speed ...etc i.e. a group of students in the campus. This model is divided into the following sub models: Random-Based, Random Walk, Random Waypoint, Random Direction and City Section. This research will use RandomBased and Random WayPoint Mobility Models:

\subsubsection{Random-Based Mobility Model (RBMM)}

In this model, the mobile node moves for choosing the destination, speed and direction randomly and freely selecting without any restrictions and independently of other nodes to be more specific [6].

\subsubsection{Random WayPoint Mobility Model (RWMM)}

The random waypoint model was proposed by "Johnson and Maltz". The mobile nodes moved randomly and their location, speed and acceleration change with the passage of time. This mobility model is simple and widely available. It is the most popular mobility models to evaluate MANETs routing protocols. Figure 3 given below shows how node moves from one waypoint $\mathrm{Pi}$ to the next $\mathrm{Pi}+1$ [7].

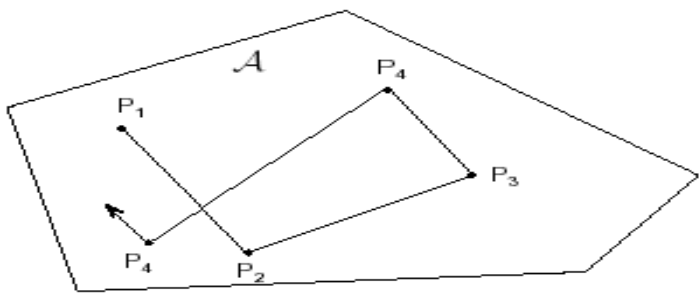

Fig 3. Node Movement in Random WayPoint Mobility Model

\section{RELATED WORK}

So far some research has been done to analyze and evaluate the performance of OLSR or comparing OLSR with other routing protocols by using different mobility models.

Plesse et al. [8] conducted research on measurement of the performance of OLSR in a military mobile ad hoc network in "URBAN" area through determining the requirement of military by using OLSR platform called "The CELAR MANET", which work in Ad-Hoc Networks for achieving a good performance.

Panda [9] implemented and compared mobility models, namely, Random WayPoint, Random Walk and Random Direction by using NS-2 simulator for analysis and to evaluate the performance of networks.

IKEDA et al. [10] evaluated the performance of MANETs under the impact of Mobility by studying a small testbed using 5-laptops to implement experiments by six OLSRMANET models.

Kulla et al. [11] performed the experiment in "Testbed" by installing specific software for OLSR and AODV on "UBUNTU 9.04 LINUX (x5)" to study the performance of OLSR and AODV under impact of the source node and the destination node movement.

Lakki et al. [12] enhanced mobility new approach for OLSR protocol by using NS-2 simulator to improve the weaknesses of the metric and exploited the information offered to improve the efficiency of the OLSR routing protocol by improvement of the three versions: the standard OLSR (Std-OLSR), Mobility-OLSR (Mob-OLSR) and OLSR-2-Mob.

Mohapatra and Kanungo [13] performed the analysis of the performance of AODV, DSR, OLSR and DSDV routing protocols by using NS-2.34 simulator. They concluded that DSR is a best in average of PDR under impact of high mobility and when the network size is less than 600x600sqm, but the PDR of OLSR is better under impact of high mobility and when the size of network is more than $600 \times 600$ sqm

Sharma and Kumar [14] performed the experimental analysis and evaluation of OLSR performance by using NS-2 simulator. They mentioned that OLSR routing protocols sending and receiving packet is $98 \%$ and less than $2 \%$ packets are wasted, so OLSR is best for large network.

Prabu and Subramani [15] analyzed the performance of modified OLSR protocol for MANETs using ESPR algorithm for evaluating the OLSR performance by using NS-2 simulator. In the results of this research, the performance of ESPR-OLSR is better as compared to the performance of the normal OLSR, because the ESPR-OLSR used a new routing 
algorithm, which perform calculation on the basis of the stability of the link and the calculation of distance and motion and, which is executed based on the concept of the edge node selection with height potential score.

All the research papers mentioned in this section discussed the experimental or actual simulation of routing protocols of MANETs by showing the measurements of the performance for example, end-to-end delay, throughput, control overhead ...etc in different environment such as military and campus on different operating systems like LINUX, UNIX, WINDOWS ...etc and by using different simulators like NS, OPNET ...etc and by changing the simulation parameters. Some researchers changed the number of nodes, simulation time and area size, while some researchers changed the parameters of nodes movement i.e mobility model, node speed and pause time. All the above mentioned used different connection pattern such as UDP, TCP ...etc with different application traffic like CBR and SINK. When OLSR was compared with AODV and DSR routing protocols, the OLSR showed better performance as compared to others. Moreover enhanced OLSR produced much better results.

\section{SIMULATION AND EVALUATION}

\subsection{Network Simulator (NS)}

Network Simulator (NS) was developed as part of the VINT (Virtual Internet Testbed) PROJECT. In 1995 NS-1 was developed, in 1996 NS-2 was developed, which than included object-oriented TCL in released from NS-1 to NS-2.35, and in NS-3. NS is an open source, which is used by the scientific community for network research as a discrete event simulator. It is possible for windows and Linux ...etc for simulating both wire and wireless networks. In this research NS-2.35 is used. It is available on "nsnam" website in zip file (NS-allinone2.35.tar.gz). There are many software, which are used with NS either included in NS or available separate. The software includes in NS are the following: NS for executing the TCL coding, NAM for showing the datagram of simulation, the trace file (.tr) is the outcomes from the simulation and are stored in it in different format such as a string, numbers, words and symbols, and Xgraph for showing the graph of simulation from the trace-file [16].

\subsection{Simulation and Analysis Software Tools}

In addition to the NS-2.35 software tools, there are some separate software tools in which some need to be configured with NS and other do not require configuration. The software tools are used for building the scenario of the simulation and others are used for analysis and drawing the graph of the measurements parameter. The simulation and analysis software tools are used in this research are the following: TCL script language [17] for building the scenario, AWK is a particular program not included in NS and there is no need to configure it with NS, it is used to generate the value of the measurements for example: throughput, End_To_End_Delay dependent on the event in the trace file such as: $r=$ "received packet", s="sent packet", $\mathrm{f}=$ "forwarded packet" and $\mathrm{m}=$ "movement node" ...etc [18] and Trace-Graph is a separate software tools Copyright (c) 2001-2005 by "Jaroslaw Malek", which is used for showing the information of the simulation in the graph, image and statistical format.

\subsection{Configuring OLSR Routing Protocol in NS-2.35}

OLSR routing protocol is not included in all version of NS-2, therefore when someone wants to work in OLSR than the researcher must build OLSR packets in NS by the UM-OLSR code and configure it. The UM_OLSR done by "Andrey Lyubimov" has been chosen for this research as it is fully complied with IETF's "RFC 3626" and supports all core functionalities of OLSR.

\subsection{Parameters for Simulation}

The parameters used for simulation in this research are summarized in table 1 which is given below.

Table 1. Simulation Parameters

\begin{tabular}{|c|c|}
\hline Operating system & Linux Mint 17.1 Cinnamon 64-bit \\
\hline Simulator & NS-2.35 \\
\hline Routing Protocol & OLSR \\
\hline Simulation Area & 1500m x1500m \\
\hline Simulation time & $40,60,80,100$ \\
\hline Number of nodes & UDP \\
\hline Connection Pattern & CBR \\
\hline Traffic type / Packet Type & 512 bytes \\
\hline Packet size & 5-10-15-20-25-30 m/s \\
\hline Node speed & $0.500000 \mathrm{~s}$ \\
\hline pause time & Random-based \\
\hline \multirow{2}{*}{ Mobility models } & Random waypoint \\
\cline { 2 - 2 } & 8 \\
\hline Total number of scenarios &
\end{tabular}

\subsection{Performance Measurement}

All the simulation in this research has been done in NS-2.35. Moreover all the simulation scenarios are carried out using OLSR routing protocol to study and evaluate the performance of OLSR by using the following measurements:

1) Sent Rate: The total number of CBR data packets, which are sent by the source node.

2) Received Rate: The total number of CBR data packets, which are received by the destination node.

3) Drop Rate: The total number of CBR data packets, which are dropped by the source or destination or any MPR nodes.

4) Average End-To-End Delay: It is the average time taken by a data packet to reach the destination.

5) Packet Delivery Ratio: It is the ratio of the data packets successfully delivered to the destination (Received Packets/Sent Packets).

6) Normalized Routing Overhead: It is the total number of routing packets divided by a total number of delivered data packets at the destination node.

7) Average Throughput: An average throughput is the average rate of packets successfully transferred to their final destination per unit time. An average throughput is calculated as;

Throughput $(\mathrm{bit} / \mathrm{sec})=$ Total of packets delivered $\times$ packet $\underline{\text { Size } \times 8}$

Total time of simulation

\subsection{Implementation Scenarios}

\subsubsection{Mobility Configuration}

There are two different mobility models used in this research, namely, Random-Based and Random WayPoint mobility models. These mobility models are used to measure the 
performance of OLSR routing protocol. The configuration of mobility to all the scenarios is as follows: The initial time of running the simulation is 0.000000 s. All nodes are deployed at the center of the simulation area, which is equal to $X=750$, $\mathrm{Y}=750$ and $\mathrm{Z}=0.0$, at $0.000001 \mathrm{~s}$. All the nodes are dispersed randomly over the area of the simulation for getting the initial position. The source node is a node number zero (node_(0)), its initial position is $X=100.43$ and $Y=880.92$. The destination node is a node number seventeen (node_(17)), its initial position is $\mathrm{X}=1200.78$ and $\mathrm{Y}=10.68$. the pause time for all nodes is started at $0.500000 \mathrm{~s}$ till $1.000000 \mathrm{~s}$ and then at $1.000000 \mathrm{~s}$ all nodes started movement at different speed (min $5.0 \mathrm{~m} / \mathrm{s}$, $\max 30.0 \mathrm{~m} / \mathrm{s}$ ), which is changing after each $5.000000 \mathrm{~s}$ till the time of simulation is completed (at 30seconds).

Table 2 : The time and speed of node, when each node change its speed

\begin{tabular}{|c|c|}
\hline Time when the nodes changed speed & Speed \\
\hline $1.000000 \mathrm{~s}$ & $30.0 \mathrm{~m} / \mathrm{s}$ \\
\hline $5.000000 \mathrm{~s}$ & $10.0 \mathrm{~m} / \mathrm{s}$ \\
\hline $10.000000 \mathrm{~s}$ & $25.0 \mathrm{~m} / \mathrm{s}$ \\
\hline $15.000000 \mathrm{~s}$ & $20.0 \mathrm{~m} / \mathrm{s}$ \\
\hline $20.000000 \mathrm{~s}$ & $15.0 \mathrm{~m} / \mathrm{s}$ \\
\hline $25.000000 \mathrm{~s}$ & $5.0 \mathrm{~m} / \mathrm{s}$ \\
\hline
\end{tabular}

\subsubsection{Mobility Generator}

\subsubsection{Sensing the Grid of the Simulation Area}

The sensing mechanism is available in NS, each node will sense all nodes in simulation one by one as the following: put "start set dist ..." for declaring that sensing is started and \$god_(is the name of God instance include the argument number of the mobile_node) set-dist from node to node in number of hops For example: "\$god_set-dist 099 6", six hops between the node number(99) and the node number(0). It is repeated for all nodes, for example in scenario at 100 nodes defined for node(0), it is repeated (99) once, but for node(99) it is not repeated, because it is repeated to node (99) with all previous nodes. After definition "set-dist" must determine in which time it will be starting as \$ns at 0.0855511013 "\$god_ set-dist 099 6", mean that, the node number (0) started sensing the grid of simulation to find the node number (99) through 6 hops at 0.0855511013 second.

\subsubsection{Tools of Mobility Generator}

There are different tools available in NS-2.35 to generate the nodes movement dependent on the main classical mobility models such as: "setdest" is a mobility tool, which is used for entity mobility models, but "grcmob" is a mobility tool, which is used for group mobility models. The performance of OLSR routing protocol is studied by using the entity mobility models, namely, RBMM and RWPMM, so in this research discussion is about the "setdest", which is used for generating the location, movement speed and direction waypoint. There are two formats for using "setdest" which are as follows:

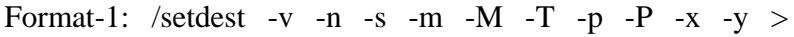
tcl_file_name.tcl, which is executed on the terminal of the operating system.

Format-2 : \$ns at movement_time "\$node_(node-no) setdest $\mathrm{X}$-target Y-target speed", which is executed on the TCL script file. This research work used "setdest" in format- 2 for the movement of nodes in all the scenarios of the simulation.

\subsubsection{Random-Based Mobility Model (RBMM) Scenarios}

All nodes moved at the same time to different target (select randomly) by the "rand" function in the procedure of movement nodes, which is applied in all scenarios of RBMM include: Defining the variable, the initial time and set the current time of the simulation and for loop from " 1 " till the total number of nodes incremented by " 1 ".

set the $\mathrm{X}$ for target and set the $\mathrm{Y}$ for target by the "rand" function and start mobility for all nodes by "setdest" at 1.000000 s to the target $X Y$ in speed at $30.0 \mathrm{~m} / \mathrm{s}$ and change the speed from range[5-10-15-20-25-30] of all nodes after $5.000000 \mathrm{~s}$ as shown in table 2 and then calling the procedure at $1.000000 \mathrm{~s}$.

All the nodes move randomly based on the "rand" function in the four scenarios dependent on the number of nodes $(40,60$, 80 and 100). The scenarios are elaborated as follows: scenario_1 at 40 nodes, scenario_2 at 60 nodes, scenario_3 at 80 nodes and scenario_4 at 100 nodes, for that four TCL script files are built. In this research, in scenario_1 at 40 nodes and scenario_ 4 at 80 nodes by NAM of them as shown in the figures given below.

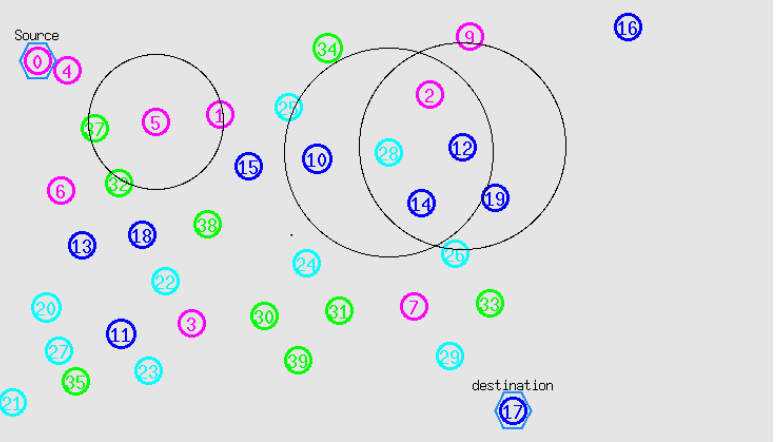

Fig 4. NAM window of RBMM Scenario_1 containing 40 Nodes

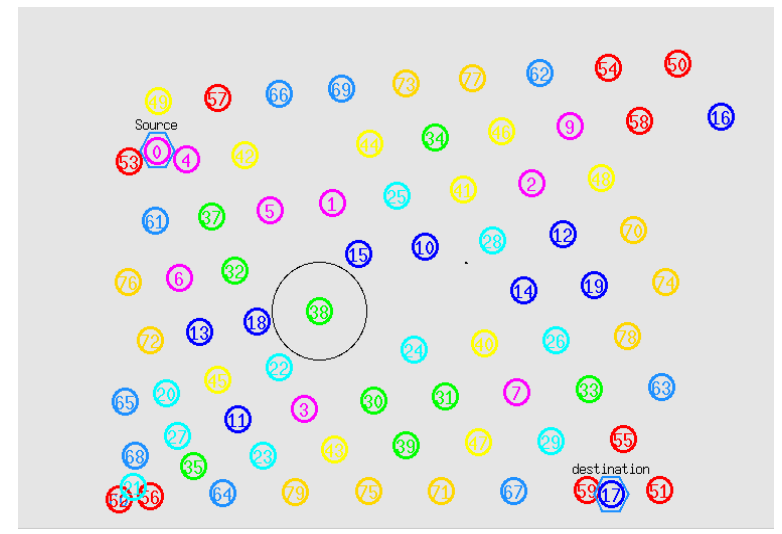

Fig 5. NAM window of RBMM Scenario_3 containing 80 Nodes

\subsubsection{Random Waypoint Mobility Model (RWPMM) Scenarios}

All the nodes moved at the same time to different target, that is determined by the "rand" function with the way point for $\mathrm{X}$ direction and $\mathrm{Y}$ direction at the speed of node with choice from range [Vmin, Vmax] in the procedure of movement nodes is applied in all scenarios of RWPMM including: 
defining the variable and the initial time and for loop from "1" till the total number of nodes incremented by " 1 ".

set the X for target and set the Y for target by the "rand" function and start mobility for all nodes by "setdest" at 1.000000 s to the target $\left(\mathrm{X} \times \mathrm{X} \_\right.$waypoint $) \&(\mathrm{Y} * \mathrm{Y}$ _waypoint $)$ in speed from range[5-10-15-20-25-30] of all nodes after $5.000000 \mathrm{~s}$ as shown in table 2 .

This process will repeat " 6 " times dependent on the speed of the movement and then calling that procedures "mob-proc$1,2,3,4,5,6$ "as $\$ \mathrm{~ns}$ at time in range [1.000000,5.000000, $10.000000,15.000000,20.000000$ and 25.000000].

All the nodes move randomly based on the "rand" function with the factor of waypoint in the four scenarios dependent on the number of nodes as the following: scenario- 5 at 40 nodes, scenario- 6 at 60 nodes, scenario- 7 at 80 nodes and scenario- 8 at 100 nodes, for this purpose four TCL script files are built in each TCL script file there are six procedures for nodes movement dependent on the speed of nodes. This research indicates scenario_6 at 60 nodes and scenario_8 at 100 nodes by NAM of them as the following:

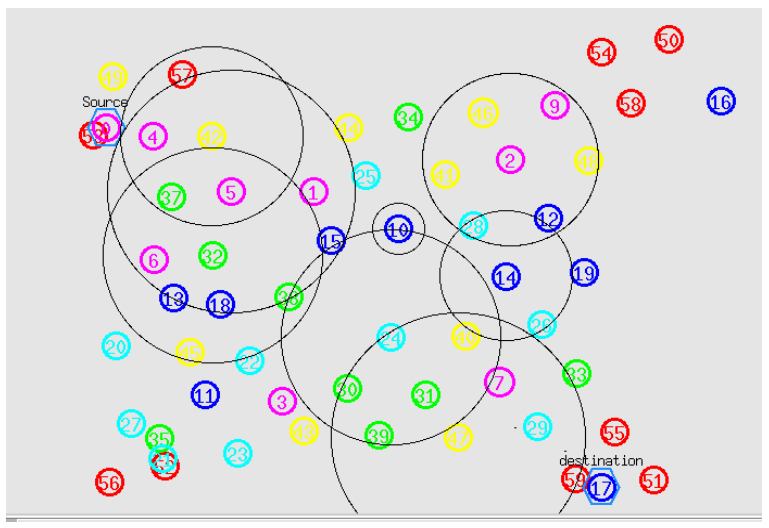

Fig 6. NAM window of RWPMM Scenario_6 containing 60 Nodes

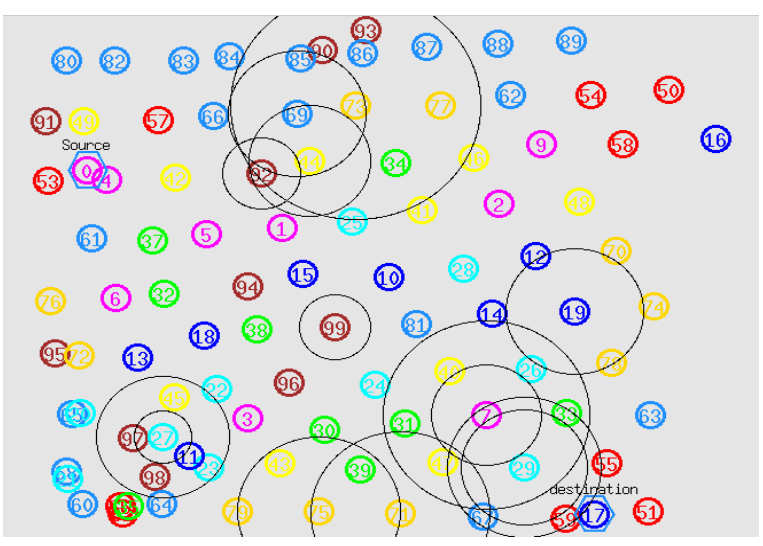

Fig 7. NAM window of RWPMM Scenario_8 containing 100 Nodes

\section{RESULTS AND DISCUSSIONS}

This section includes the results and graphs for evaluating the performance of OLSR routing protocol. There are two main scenarios based on mobility models, namely, Random-Based Mobility Model (RBMM) and Random WayPoint Mobility Model (RWPMM). Moreover there are four sub scenarios for each main scenario. The value of the measurements of performance is calculated from the trace file based on the event by using Xgraph, Trace Graph and the AWK language script. Also the graph of the measurements is generated by Xgraph, Trace Graph and Microsoft Excel.

\subsection{Sent Rate}

Fig 8 shows the sent rate of CBR data packets. In the case of 40 nodes and 100 nodes, the source node under RBMM sent CBR data packets are more than the source node under RWPMM, but at 60 nodes and at 80 nodes, the source node under RWPMM sent CBR data packets are more than the source node under RBMM, therefore the performance of OLSR under impact of RWPMM is better than under RBMM, because the source node under RWPMM sent CBR data packets are more than the source node under RBMM.

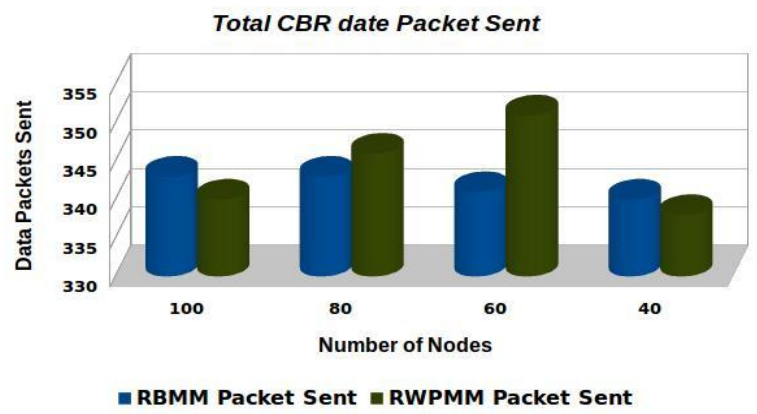

Fig 8. Sent Rate of CBR data packet, comparison of RBMM and RWPMM

\subsection{Received Rate}

Fig 9 shows the received rate of CBR data packets. In the case of 40 nodes, the destination node under RBMM received CBR data packets are more than the destination node under RWPMM, but at 60 nodes, 80 nodes and 100 nodes, the destination node under RWPMM received CBR data packets are more than the destination node under RBMM, therefore the performance of OLSR under impact of RWPMM is better than under RBMM, because the destination node under RWPMM received CBR data packets are more than the destination node under RBMM

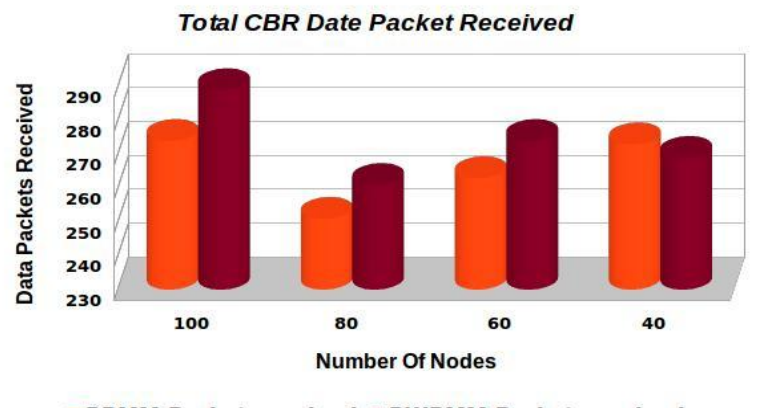

Fig 9. Received Rate of CBR data packets, comparison of RBMM and RWPMM

\subsection{Drop Rate}

Fig 10 shows the drop rate of CBR data packets. The packets are dropped by the source node or destination node or any MPR nodes due to reasons such as, buffer overflow, no MPR nodes for forwarding the data packets, due to the network of OLSR is not complete built, some MPR nodes lost its location by mobility and waiting for selecting another nodes replace it ...etc. In the case of 40 nodes, the OLSR network under RBMM and RWPMM dropped CBR data packets at 20\%, also at 60 nodes dropped data packets are $22 \%$, so the network 
of OLSR dropped the same CBR data packets under both RBMM and RWPMM, but at 80 nodes, the network of OLSR dropped CBR data packets at $25 \%$ in RBMM, but under RWPMM at 24\%, also the network of OLSR dropped CBR data packets at $17 \%$ under RBMM, but under RWPMM at $12 \%$, so that the network of OLSR under RBMM dropped CBR data packets more than the network of OLSR under RWPMM, therefore the performance of OLSR under impact of RWPMM is better than RBMM, because the OLSR network under RWPMM dropped data packets less than under RBMM.

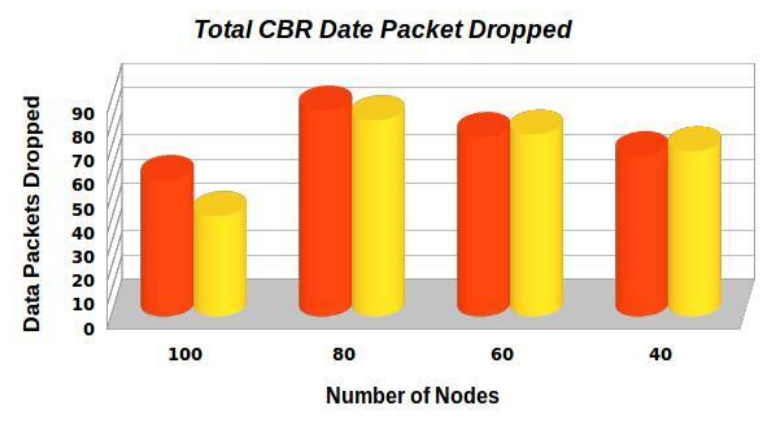

- RBMM Packet drop $\approx$ RWPMM Packet drop

Fig 10. Drop Rate of CBR data packet, comparison of RBMM and RWPMM

\subsection{Average End-To-End Delay}

Fig 11 shows the average end to end delay in seconds. In all scenarios $(40,60,80$ and 100 nodes $)$, the average end to end delay of the OLSR network under RBMM is less than the OLSR network under RWPMM, therefore the performance of OLSR under impact of RBMM is more efficient as compared to the performance of OLSR under RWPMM.

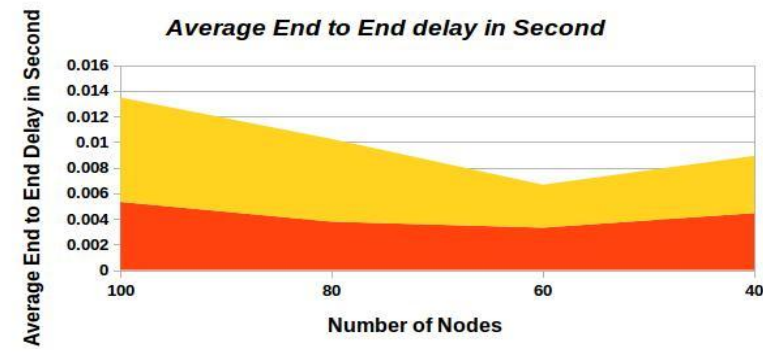

= RBMM Average End to End Delay $=$ RWPMM Average End to End Delay

Fig 11. Average End to End Delay in seconds, comparison of RBMM and RWPMM

\subsection{Packet Delivery Ratio}

Fig 12 shows packet delivery ratio of CBR data packet. In the case of 100 nodes, 80 nodes and 60 nodes, the network of OLSR under RWPMM is more efficient than the network of OLSR under RBMM, but in case of 40 nodes, the network of OLSR under RBMM is more efficient than the network of OLSR under RWPMM, therefore the performance of OLSR under impact of RWPMM is better than under RBMM, because the packet delivery ratio under RWPMM is more than under RBMM.

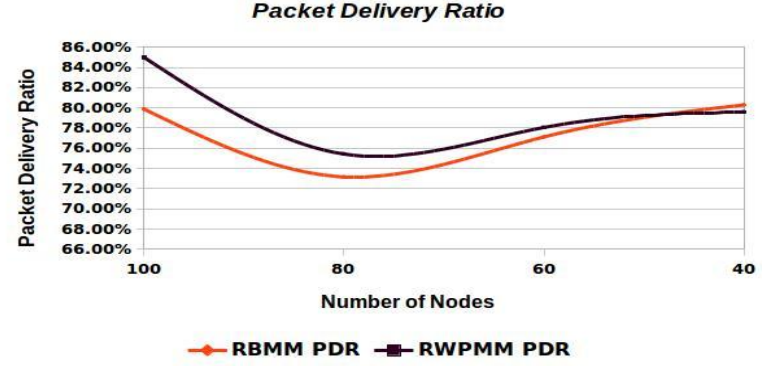

Fig 12. Packet Delivery Ratio comparison of RBMM and RWPMM

\subsection{Normalized Routing Overhead}

Fig 13 shows normalized routing overhead. In all scenarios (40,60, 80 and 100 nodes), the overhead of the network of OLSR under RWPMM is less than the overhead of the network of OLSR under RBMM, therefore the performance of OLSR under impact of RWPMM is better as compared to the performance of OLSR under RBMM, because the OLSR overhead under RWPMM is less than under RBMM.

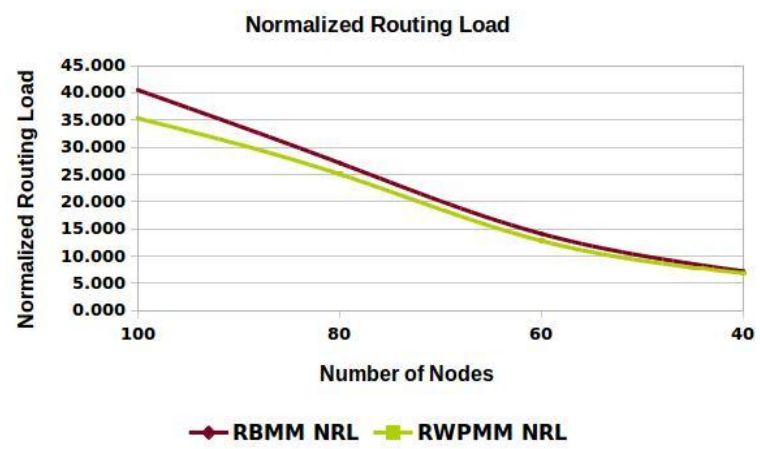

Fig 13. Normalized Routing Overhead, comparison of RBMM and RWPMM

\subsection{Average Throughput}

Fig 14 shows average throughput (bit/sec). In the case of 40 nodes, the average throughput of the network of OLSR under RBMM is more than the network of OLSR under RWPMM, but in the case of 100 nodes, 80 nodes and 60 nodes, the average throughput of the network of OLSR under RWPMM is more than the throughput of the OLSR network under RBMM, therefore the performance of OLSR under impact of RWPMM is better than the performance of OLSR under RBMM, because the average throughput under RWPMM is more than under RBMM. 


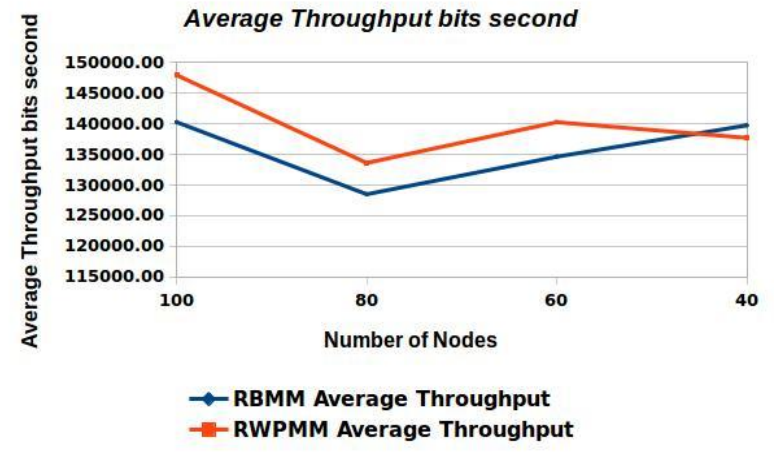

Fig 14. Average Throughput (bit/sec), comparison of RBMM and RWPMM

\section{CONCLUSION AND FUTURE WORK 5.1 Conclusion}

This research provides an evaluation of the performance of OLSR routing protocol by using different mobility models, namely, random-based mobility model and random waypoint mobility model by using NS-2.35.

In general, the mobility of the mobile ad hoc networks (MANETs) is a major issue, because when the mobile node changes its target by mobility, the topology of the network would change, also all the information about nodes in the network, which is stored in routing table and other tables will also change, because the route between the source node and destination node is changing continuously, which means the MPR nodes are frequently changing, and as a result the OLSR network do not build and some data packets are dropped.

In random-based mobility model each node change its target once at $0.500000 \mathrm{~s}$, that mean during simulation at $30 \mathrm{~s}$, each node changed its target 60 times dependent on the mathematics of the "rand" function, which generates values between ( 0 and 3 ) only and nothing else, so all nodes move across the destination node, but in random waypoint mobility model, each node changed its target dependent on the mathematics of the "rand" function with the factor of the way point was determined in the simulation and changed at the time started at $1.000000 \mathrm{~s}$ and than changed after $5.000000 \mathrm{~s}$ till the time of simulation finish, so the mobile nodes come near to the destination node or in the center of the simulation area.

Also for the applications traffic of CBR data packets, the mobility in delay to build the OLSR network, so that the application traffic of CBR data packets experience delay between $0.5 \mathrm{~s}$ and $4.8 \mathrm{~s}$ instead of the time of start the application traffic of CBR data packets at 5.0s, so there are so many CBR data packets dropped, because the MPR nodes between a source and a destination has not yet completed selecting them.

The number of sent, received and dropped data packets change, when the TCL script file runs more than one time and it leads to change the value of all the measurements of the simulation, because the value is generated by the "rand" function is changed.

In short, the performance of OLSR by using RWPMM is more efficient as compared to the performance of OLSR by using RBMM, because the most measurements gave batter result under RWPMM than under RBMM.

\subsection{Future Work}

The mobility is a major issue in MANETs, so there are two categories dependent on the movement of nodes alone or together, namely, entity mobility models and group mobility models each one include so many models which has been discussed in the introduction section. This research focused on two entity mobility model such as RBMM and RWPMM. There are still many issues which are not yet properly addressed by the research community. These are as follows:

- Avoiding the movement of mobile nodes around the destination node or in the center of the simulation area, when the time of simulation is increased.

- $\quad$ Avoiding the stopping of the mobile node around 2 seconds in the edge of the simulation area when any node reached there.

- Avoiding the simulation error message, when the way point direction with the value of "rand" is more/less than the area of simulation.

- $\quad$ Study the performance of OLSR routing protocol by using two or more than two mobility model in the same scenarios.

- In the large simulation area having few number of mobile nodes, how to increase the range of radio frequency for nodes to communicate with the far node in the limited time of simulation.

\section{REFERENCES}

[1] Gupta, R., 2011. "Mobile adhoc network (MANETS): Proposed solution to security related issues. Indian Journal of Computer Science and Engneering (IJCSE), 2(5), pp.738-746.

[2] Lin, T., 1999. Mobile ad-hoc network routing protocols: methodologies and applications.

[3] Abolhasan, M., Wysocki, T. and Dutkiewicz, E., 2004. A review of routing protocols for mobile ad hoc networks. Ad hoc networks, 2(1), pp.1-22

[4] Clausen, T., Jacquet, P., Adjih, C., Laouiti, A., Minet, P., Muhlethaler, P., Qayyum, A. and Viennot, L., 2003. Optimized link state routing protocol (OLSR).

[5] Camp, T., Boleng, J. and Davies, V., 2002. A survey of mobility models for ad hoc network research. Wireless communications and mobile computing, 2(5), pp.483502 .

[6] Bai, F. and Helmy, A., 2004. A survey of mobility models. Wireless Adhoc Networks. University of Southern California, USA, 206.

[7] Yoon, J., Liu, M. and Noble, B., 2003, March. Random waypoint considered harmful. In INFOCOM 2003. twenty-second annual joint conference of the IEEE computer and communications. IEEE societies (Vol. 2, pp. 1312-1321). IEEE.

[8] Plesse, T., Adjih, C., Minet, P., Laouiti, A., Plakoo, A., Badel, M., Muhlethaler, P., Jacquet, P. and Lecomte, J., 2005. OLSR performance measurement in a military mobile ad hoc network. Ad Hoc Networks, 3(5), pp.575588 .

[9] Panda, S., 2009. Implementation and Comparison of Mobility Models In Ns-2 (Doctoral dissertation, National Institute of Technology Rourkela). 
[10] Ikeda, M., Hiyama, M., Barolli, L., Xhafa, F. and Durresi, A., 2010, February. Mobility effects on the performance of mobile ad hoc networks. In Complex, Intelligent and Software Intensive Systems (CISIS), 2010 International Conference on (pp. 230-237). IEEE.

[11] Kulla, E., Ikeda, M., Barolli, L., Miho, R. and Kolic, V., 2010, September. Effects of source and destination movement on MANET performance considering OLSR and AODV protocols. In Network-Based Information Systems (NBiS), 2010 13th International Conference on (pp. 510-515). IEEE

[12] Lakki, N., Ouacha, A., Habbani, A., El Khaddar, M.A., El Koutbi, M. and El Abbadi, J., 2012. A New Approach for Mobility Enhancement of OLSR Protocol. International Journal of Wireless \& Mobile Networks, 4(1), p.117.

[13] Mohapatra, S. and Kanungo, P., 2012. Performance analysis of AODV, DSR, OLSR and DSDV routing protocols using NS2 Simulator. Procedia Engineering, 30, pp.69-76.
[14] Sharma, A. and Kumar, E.R., 2013. Experimental Analysis of OLSR Routing Protocol and Performance Evaluation Using NS-2 Simulator. network, 3(5), pp.676681.

[15] Prabu, K. and Subramani, A., 2014, February. Performance analysis of modified OLSR protocol for MANET using ESPR algorithm. In Information Communication and Embedded Systems (ICICES), 2014 International Conference on (pp. 1-5). IEEE.

[16] Fall, K. and Varadhan, K., 2011. The ns Manual (formerly ns Notes and Documentation). The VINT project, 47.

[17] Ousterhout, J.K., 1993. TCL and the TK Toolkit. Computer Science Division, Department of Electrical Engineering and Computer Science, University of California, Berkeley.

[18] Close, D.B., Robbins, A.D., Rubin, P.H., Stallman, R. and van Oostrum, P., 1995. The AWK Manual. 